\title{
Estrogen deficiency leads to telomerase inhibition, telomere shortening and reduced cell proliferation in the adrenal gland of mice
}

\author{
Sharyn Bayne ${ }^{1}$, Margaret EE Jones ${ }^{2}, \mathrm{He} \mathrm{Li}^{1}$, Alex R Pinto ${ }^{1}$, Evan R Simpson², Jun-Ping Liu ${ }^{1}$ \\ ${ }^{1}$ Department of Immunology, Central Eastern Clinical School, Monash University, Melbourne, Australia; ${ }^{2}$ Prince Henry's Institute \\ of Medical Research, Melbourne, Australia
}

Estrogen deficiency mediates aging, but the underlying mechanism remains to be fully determined. We report here that estrogen deficiency caused by targeted disruption of aromatase in mice results in significant inhibition of telomerase activity in the adrenal gland in vivo. Gene expression analysis showed that, in the absence of estrogen, telomerase reverse transcriptase (TERT) gene expression is reduced in association with compromised cell proliferation in the adrenal gland cortex and adrenal atrophy. Stem cells positive in c-kit are identified to populate in the parenchyma of adrenal cortex. Analysis of telomeres revealed that estrogen deficiency results in significantly shorter telomeres in the adrenal cortex than that in wild-type (WT) control mice. To further establish the causal effects of estrogen, we conducted an estrogen replacement therapy in these estrogen-deficient animals. Administration of estrogen for 3 weeks restores TERT gene expression, telomerase activity and cell proliferation in estrogen-deficient mice. Thus, our data show for the first time that estrogen deficiency causes inhibitions of TERT gene expression, telomerase activity, telomere maintenance, and cell proliferation in the adrenal gland of mice in vivo, suggesting that telomerase inhibition and telomere shortening may mediate cell proliferation arrest in the adrenal gland, thus contributing to estrogen deficiency-induced aging under physiological conditions.

Keywords: estrogen, telomerase, telomeres, cell proliferation, aromatase knockout mice

Cell Research (2008) 18:1141-1150. doi: 10.1038/cr.2008.291; published online 21 October 2008

\section{Introduction}

Estrogen plays an important role in regulating cell proliferation in a tissue-specific manner. Estrogen shortage induces aging and atrophic changes in a number of estrogen-responsive organs including uterus, breast and brain. On the other hand, increased supplies and actions of estrogen have been implicated as playing a pivotal role in stimulating cell proliferation during tumorigenesis and progression of breast cancer and endometrial cancer [1]. Although the mechanisms by which estrogen regulates cell

Correspondence: He Li ${ }^{\mathrm{a}}$, Jun-Ping Liu ${ }^{\mathrm{b}}$

Department of Immunology, AMREP, Monash Medical School, Commercial Road, Prahran, Victoria 3181, Australia.

Tel: +61-3-99030715; Fax: +61-3-99030120

aE-mail: he.li@med.monash.edu.au

bE-mail: jun-ping.liu@med.monash.edu.au

Received 15 January 2008; revised 26 February 2008; accepted 19 March 2008; published online 21 October 2008 proliferation and aging remain to be fully understood, several genes that control cell proliferation have been shown to be downstream targets of estrogen action [2]. Among these genes, the proto-oncogene c-myc has been reported as a direct target gene of estrogen in estrogen receptor-positive cells [3]. Recently, studies have shown that estrogen stimulates the activity of the enzyme telomerase and gene expression of telomerase reverse transcriptase (TERT) in cancer cell cultures (reviewed in $[4,5])$. Telomerase is required to maintain the structures of chromosomal ends (telomeres) for continuous cell division in human embryonic development and cancer (reviewed in [6-8]). Telomerase interacts with telomeric DNA and other telomere binding proteins, catalyzing telomeric DNA reverse transcription to lengthen telomeres and capping the telomere ends [911]. In the absence of telomerase, telomeres shorten, and short telomeres trigger cell senescence and apoptosis at a threshold of telomere length, thereby limiting cell proliferative lifespan. While telomerase is consistently repressed during cell differentiation to mature somatic cells in man, 
telomerase is active in a number of somatic tissues in mice. However, little is known as to how telomerase activity is regulated in vivo under physiological conditions.

Transcriptional activation of the telomerase catalytic subunit TERT is an initial rate-limiting step for producing telomerase activity [6]. Regulated by multiple factors, the human TERT gene promoter contains a number of binding sites for transcriptional regulation $[12,13]$. There are two typical E-boxes and several GC-boxes for the transcription factors c-myc and Sp1, respectively [14-16]. The proto-oncogene c-myc is a downstream target of several growth factors and the steroid hormone estrogen [3, 17-19]. Estrogen receptor responsive element (ERE) is present in human but not mouse TERT promoter. As suggested from in vitro cell culture experiments in human vascular endothelial cells [20], ovary epithelial cells [21] and breast cancer cells [22], estrogen regulates telomerase activity possibly by direct action on the TERT gene or by indirect action through c-myc. Whether or not estrogen regulates telomerase activity in vivo and to what extent estrogen regulates cell development, aging and tumorigenesis by modulating telomerase activity in estrogen-responsive tissues such as brain, breast and ovary epithelial cells are unknown and require further investigation.

The present study was undertaken to characterize telomerase activity in a number of tissues of mice in which estrogen production is eliminated by disrupting the gene encoding the enzyme aromatase that is essential for estrogen synthesis. We show that estrogen is required for the maintenance of telomerase activity selectively in the adrenal gland of mice. Depletion of estrogen results in significant inhibition of telomerase activity and retardation of development of the adrenal gland. Hormone replacement therapy for 3 weeks reverses the estrogen deficiency-induced inhibition of telomerase activity and restores cell proliferation in the adrenals. Thus, this study shows for the first time that estrogen plays a major role in up-regulating TERT gene expression and telomerase activity in vivo; deprivation of estrogen instigates telomerase inhibition, accompanied by compromised cell proliferation and tissue atrophy in the adrenal gland.

\section{Results}

Requirement of estrogen for telomerase activity in the adrenal gland

In mice, a number of normal tissues express detectable levels of telomerase activity [23-25]. To determine whether telomerase is subjected to regulation by estrogen, telomerase activity was analyzed in a range of tissues and compared between age-matched WT mice and estrogen-deficient aromatase knockout (ArKO) mice. When telomerase activity from WT mice was compared against the telomerase-positive HeLa cervical cancer cell line, high levels of telomerase activity were detected in the liver, adrenal gland, thymus and ovary; moderate levels of telomerase activity were observed in the kidney; low levels were present in the pancreas, spleen, lung and uterus; and no telomerase activity was detected in the brain, skeletal muscle, heart and fat. There was no significant difference in telomerase activity in these tissues assayed between WT and estrogen-deficient ArKO mice, except the adrenal gland (data not shown). The adrenal gland showed detectable levels of telomerase activity in WT mice. However, significantly decreased telomerase activity was observed in estrogen-deficient ArKO mice compared with that of WT littermates. By quantifying the relative production of $\left[{ }^{32} \mathrm{P}\right]$-labeled telomeric DNA as counts per minute (CPM), we found that telomerase activity in the adrenals of ArKO mice was approximately $30 \%$ of that in the adrenals of WT animals (Figure 1).

To determine whether the reduction of telomerase activity in the estrogen-deficient mice is caused by estrogen deficiency and could be reversed by estrogen replacement therapy, approximately 10 -week-old ArKO mice were treated with exogenous $17 \beta$-estradiol (E2) for 21 days. Age-matched WT mice also received the same E2 treatment as control. In all ArKO mice tested, E2 treatment restored telomerase activity to levels equal to that of untreated WT mice. As shown in Figure 1, after E2 treatment, the levels of telomerase activity in the adrenals of ArKO mice became similar to that of WT control and much higher than that in the adrenals of ArKO mice without treatment, representing about four-fold increases (Figure 1).

\section{Reduced gene expression of the TERT in estrogen-deficient mice}

To determine the mechanisms of telomerase inhibition in the adrenal glands of estrogen-deficient mice, we assessed the gene expression levels of TERT and the transcription factor c-myc in the adrenal glands of WT and ArKO animals by RT-PCR. The housekeeping gene GAPDH was measured as a control for input and to normalize data for each gene (Figure 2). Consistent with high telomerase activity, expression of the TERT gene was high in the adrenals of WT mice. However, TERT gene expression was significantly decreased in the adrenals of ArKO mice, with approximately $60 \%$ reduction compared with that in WT adrenals $(P<0.05)$. The level of c-myc gene expression was also reduced in ArKO mice, by approximately $30 \%$ compared with that in WT adrenals $(P<0.05)$. To determine if estrogen reverses the reduced gene expressions of TERT and c-myc, a hormone replacement therapy was conducted in these WT and ArKO animals, followed by gene expres- 


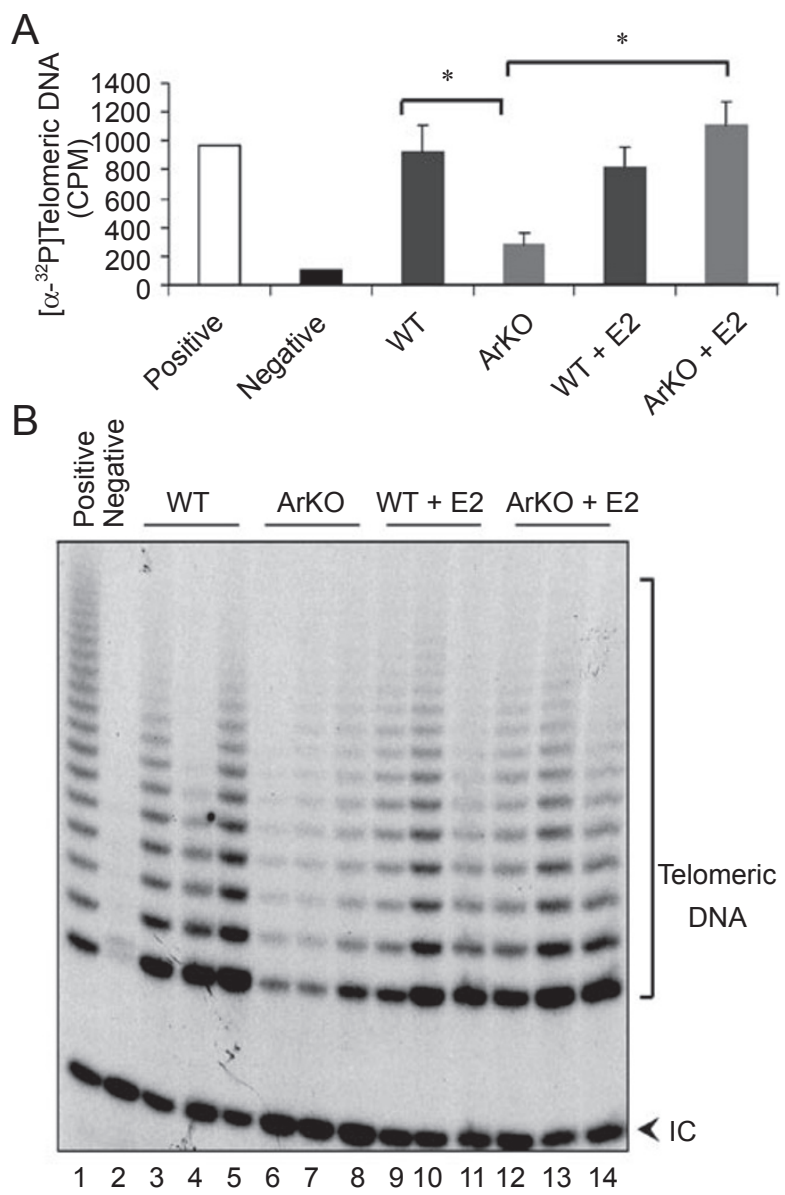

Figure 1 Telomerase activity is inhibited in the adrenal gland of ArKO mice, and inhibition is reversed by estrogen replacement therapy. (A) Quantification of telomerase activity. Telomerase activity was quantified by measuring the amount of $\left[\alpha{ }^{32} P\right]-d A T P$ incorporated into telomeric DNA in a $\beta$-counter and expressed as CPM. Samples of the adrenal tissues from estrogen-treated and untreated groups are indicated. Data are presented as mean \pm SE from at least three mice per group. $* P<0.05$. (B) Representative image of telomerase activity in the adrenal gland of wild-type (WT) and ArKO mice with or without treatment with E2, as determined by TRAP (Materials and Methods). E2 treatments were conducted for 3 weeks as also described in Materials and Methods. IC: internal control.

sion analysis for TERT and c-myc. Consistent with the responsive changes in telomerase activity, E2 replacement therapy for 3 weeks restored gene expression levels of both TERT and c-myc in ArKO adrenals to similar expression levels observed in WT adrenals (Figure 2). These data indicate a causative up-regulation of TERT and c-myc gene expressions by estrogen in the adrenals of mice in vivo.

\section{Association of telomerase inhibition with compromised cell proliferation and tissue growth of the adrenal gland}

Both estrogen and telomerase are involved in stimulat- ing cell proliferation. To investigate if loss of estrogen and inhibition of telomerase activity in ArKO mice may affect the levels of cell proliferation in the adrenal gland, we attempted to determine the status of cell proliferation by staining for the incorporation of BrdU. Despite success in other control tissues, very few BrdU-positive cells were observed and many sections from WT adrenal glands showed no BrdU labeling (data not shown). The lack of BrdU-positive cells contradicted previous reports of cell proliferation in the adrenal gland [26, 27], and we assumed that the poor BrdU uptake was due to the short delivery time of BrdU ( $2 \mathrm{~h}$ prior to tissue collection) compared with other adrenal gland studies that typically used a mini-osmotic pump delivery system over a 7 -day period $[26,28]$.
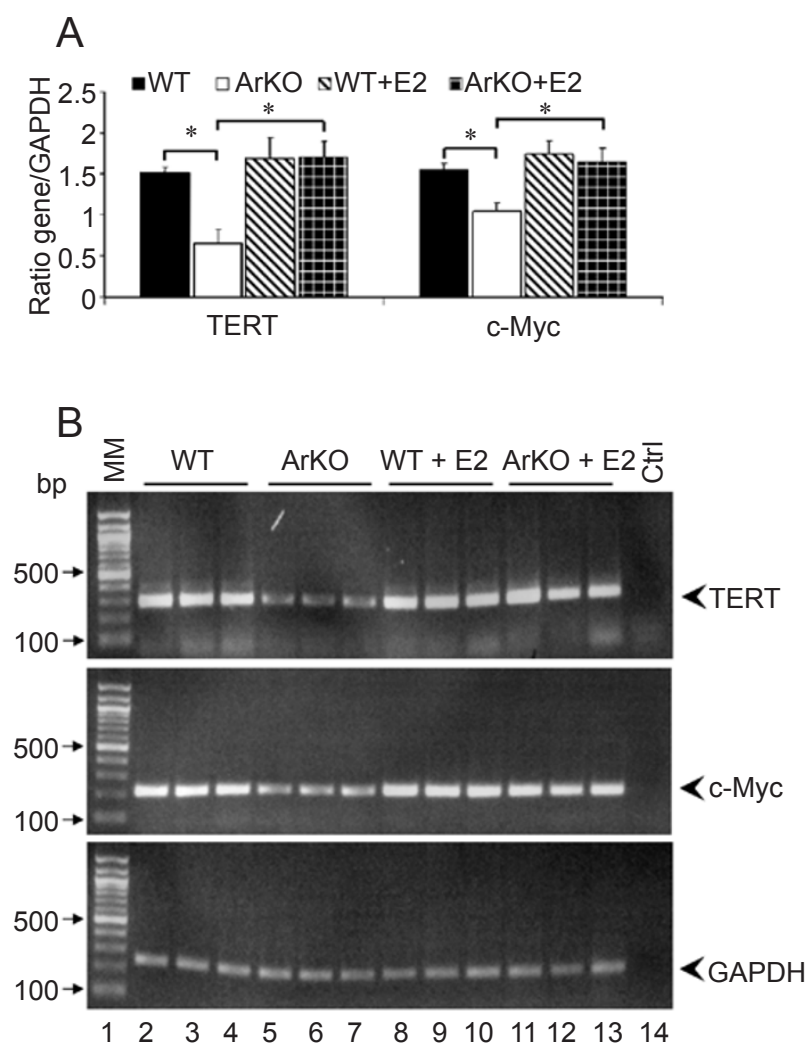

Figure 2 Decreased gene expressions of TERT and c-myc in the adrenal gland of ArKO mice and restoration by E2 treatment. (A) Gene expression levels of TERT and c-myc in wild-type (WT) and ArKO mice with or without E2 treatment as indicated. The mRNAs of TERT and c-myc in each sample were measured by RT-PCR followed by densitometry, and adjusted to GAPDH expression levels to normalize for input. The data are expressed as the mean \pm SE (ratio of gene/GAPDH). $* P<0.05$. (B) Representative images of gene expressions of TERT, c-myc and GAPDH in individual adrenal glands of WT and ArKO mice with or without E2 treatment, as measured by RT-PCR. The base pair (bp) was indicated for the lane labeled molecular markers (MM), to verify the correct size of each PCR product. A diluent only sample was included as a negative control (Ctrl). 
To overcome this problem, adrenal gland sections were stained with a specific antibody for the Ki67 protein that is expressed during all stages of the cell cycle, but repressed in $\mathrm{G}_{0}$ [29]. With specific antibodies, Ki67-positive cells were observed in adrenal gland sections from both WT and estrogen-deficient ArKO mice. These Ki67-positive cells predominantly localized at the cortex with majority of the cells in the outer area of the capsule and low levels of staining close to the cortical-medullar junction (Figure 3A). However, the numbers of Ki67-positive cells in ArKO mice appeared to be significantly less than that in the adrenal gland of WT mice $(P<0.05)$ (Figure 3A), suggesting that there is a significant reduction in proliferative cells in the adrenals of estrogen-deficient mice. To determine if the reduced number of proliferative cells can be recovered by hormone replacement therapy, we treated the estrogen-deficient ArKO mice with E2 for 21 days. As shown in Figure $3 \mathrm{~B}$ and $3 \mathrm{C}$, estrogen treatment stimulated cell proliferation in ArKO mice to levels similar to WT controls. In addition, comparing the average size and weight between WT and
A
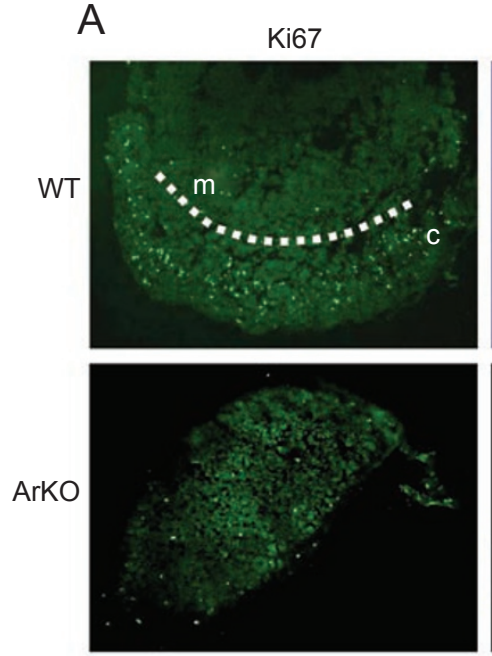

DNA
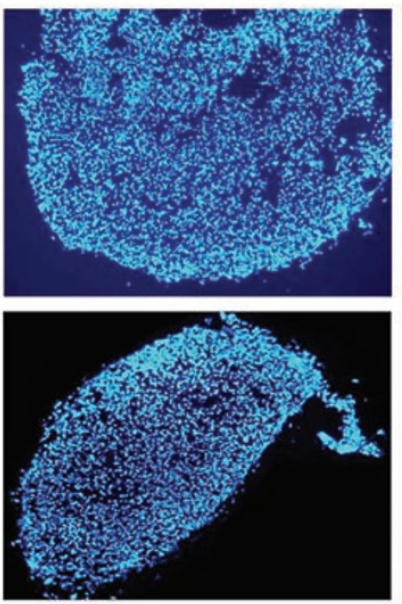

Merge
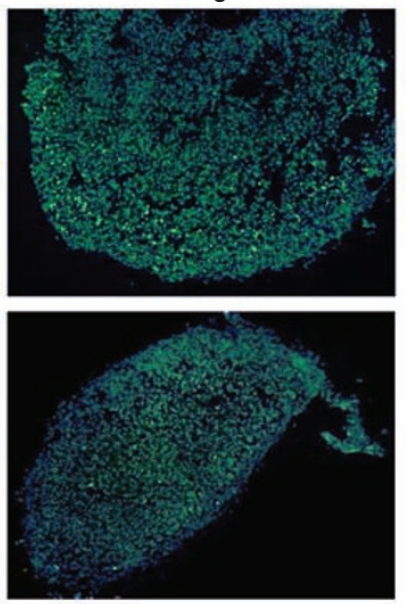

B

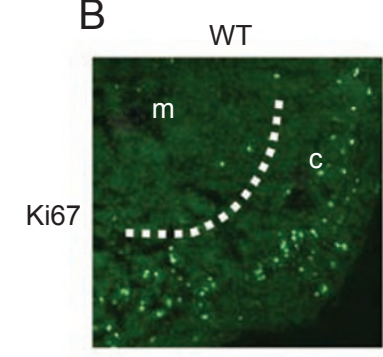

C
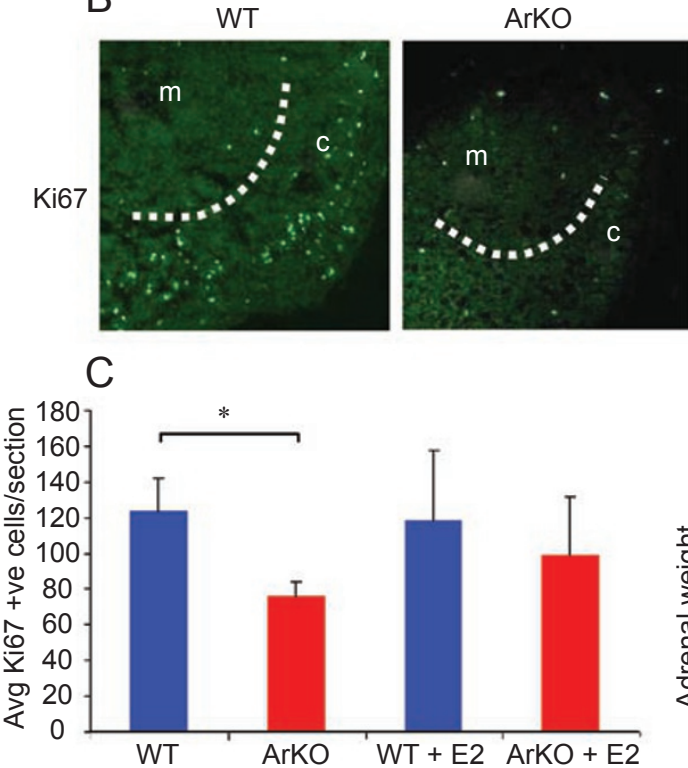

$\mathrm{WT}+\mathrm{E} 2$

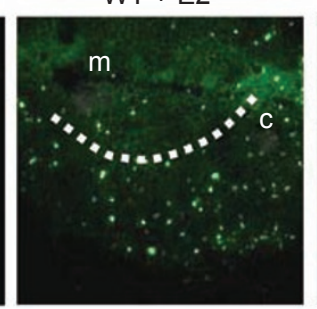

$\mathrm{D}$
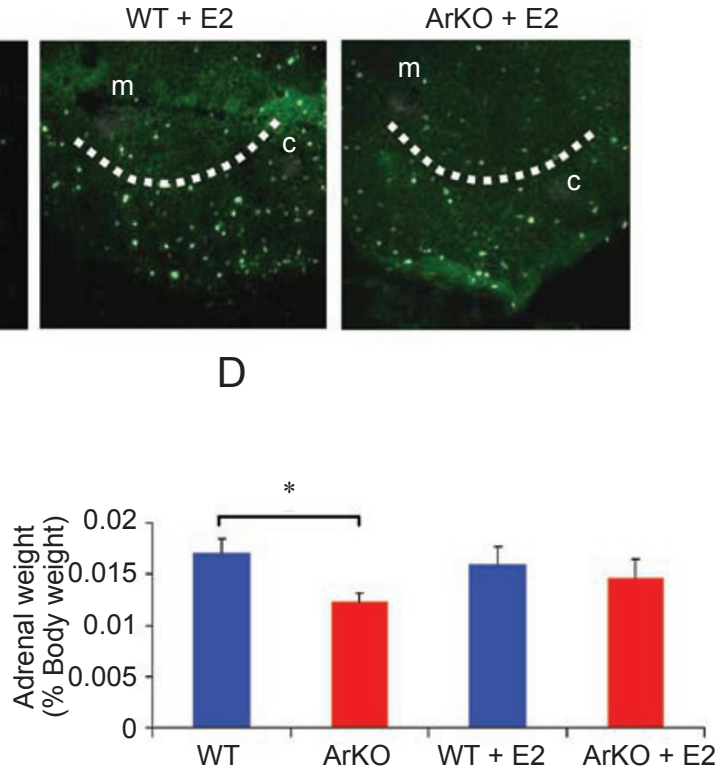

Figure 3 Estrogen deficiency inhibits cell proliferation and tissue growth in the adrenals of ArKO mice. Tissue sections were stained for expression of the cell proliferation marker Ki67. Triplicate sections were stained for three to five mice per group. (A) Representative images from untreated WT and ArKO mice. Ki67 staining (green spot), nuclear staining (blue) and merged staining signals are shown. m, medulla. c, cortex. (B) Ki67 staining of the adrenal cortex sections from WT and ArKO mice treated with estrogen (E2) for 3 weeks. m, medulla. c, cortex. (C) Quantitative data were expressed as the average (Avg) number of positive cells \pm SE per section. (D) Average wet adrenal weight \pm SE, expressed as a percentage of total body weight, from three to five mice per group, ${ }^{*} P<0.05$. 
ArKO, we found that the size and weight of the adrenal gland in the estrogen-deficient ArKO mice were significantly less than those of WT littermates (Figure 3A), and E2 replacement therapy reversed the weight reduction of the adrenal gland in ArKO mice (Figure 3D).

\section{Telomere shortening in the adrenal cortex of estrogen- deficient mice}

To determine the impact of estrogen deficiency on telomeres, and the consequence of estrogen deficiency-induced telomerase inhibition in ArKO mice, we analyzed the sizes of telomeres in the adrenal gland of these mice with or without estrogen in vivo. By quantitative fluorescence in situ hybridization (Q-FISH) using specific fluorescencelabeled telomeric DNA probe, we found for the first time that telomeres in the cells localized in the adrenal cortex are significantly longer than those in the cells of adrenal medulla (Figure 4, panel C versus panel E). This finding was initially surprising, but was consistent with the data that proliferating cells mainly populated in the cortex of the adrenal gland (Figure 3). Comparing the telomere lengths in the adrenal medulla between the wild-type and ArKO mice showed no significant difference (Figure 4, panel E versus F; panel G). However, striking differences in telomere lengths emerged in the adrenal cortex between wild-type and ArKO mice (Figure 4, panel C versus D; panel G). While the telomere length in the adrenal medulla was approximately $40 \%$ of that in the cortex in both wildtype and ArKO, the telomere length in the adrenal cortex of ArKO mice was reduced to be indistinguishable from the telomeres in the medulla (Figure 4G). The telomere length decreased by $60-70 \%$ in the ArKO adrenal cortex compared with that in the wild type, showing a significant correlation with the reduced telomerase activity and TERT gene expression (Figures 1 and 2) and with the loss of proliferative cells in the adrenal cortex (Figure 3).

\section{Identification of proliferative and stem cell populations}

Increasing evidence suggests that the adrenal gland contains a population of stem/progenitor cells and there are different patterns in the proliferation, differentiation and migration of these cells [30]. We examined whether the proliferative cells identified by Ki67 staining express the stem cell markers stem-cell antigen-1 (Sca-1) and/or c-kit, two markers that are commonly used to identify stem cell populations [31,32]. Expression of Sca-1 and c-kit was examined by immunohistochemical staining of WT adrenal gland sections and co-localization with proliferating cells was determined by double staining for Ki67 expression. Since neither Sca-1 nor c-kit expression has been reported in adult mouse adrenal glands, we performed positive controls using the sections of thymus [33] and ovary [34]
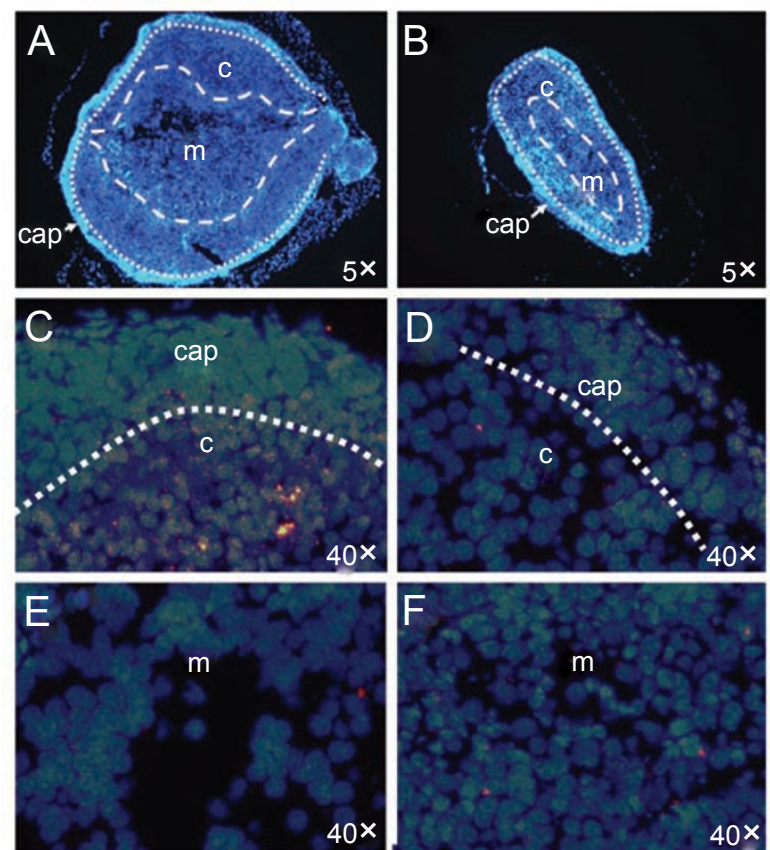

G

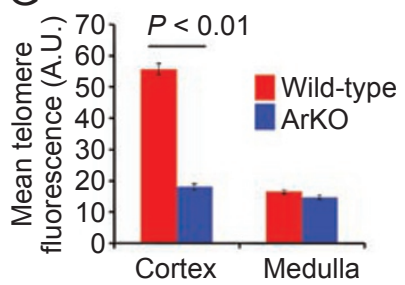

Figure 4 Estrogen deficiency induces telomere shortening in adrenal cortex cells. Adrenal glands from wild-type (WT) and ArKO mice were sectioned at $\sim 5 \mu \mathrm{m}$ thickness and examined for telomeres by quantitative fluorescence in situ hybridization (Q-FISH) (Materials and Methods). Chromosomal DNA in the adrenal tissue sections of WT (A) and ArKO (B) was stained with DAPI. Borders of adrenal cap, cortex (c) and medulla $(\mathrm{m})$ are indicated by dotted lines. Illustrative figures of merged micrographs for telomere $\mathrm{Q}$ FISH showing telomeres (red dots) and DNA (blue) of WT cortex and medullar cells ( $\mathbf{C}$ and $\mathbf{E}$, respectively), and ArKO cortex and medullar cells ( $\mathbf{D}$ and $\mathbf{F}$, respectively). (G) Histogram of quantified mean telomere fluorescence intensity ( \pm S.E.M.) of at least 50 cells randomly chosen for measurement from WT and ArKO cortex and medulla cells. $P$-value represents the difference of the mean telomere lengths between WT and ArKO mice.

tissues where the expression of Sca-1 has been reported [33]. Although it was readily detected in the thymus, Sca-1 was not detectable in the adrenal gland (data not shown). However, c-kit was detected in a restricted region of the adrenal cortex of WT mice (Figure 5). Despite that both c-kit and Ki67 were expressed predominantly within the regions of the adrenal cortex, there was limited number of co-stained cells positive for both Ki67 and c-kit (Figure 5). Furthermore, c-kit-positive cells appeared to be populated 
more cortical than Ki67-positive cells (Figure 5). These data suggest that there are at least four distinct cell populations: c-kit-positive, Ki67-positive, double-positive and double-negative cells in adrenal cortex. Estrogen deficiency may thus mainly affect c-kit-negative Ki67-positive pro-

\section{A}
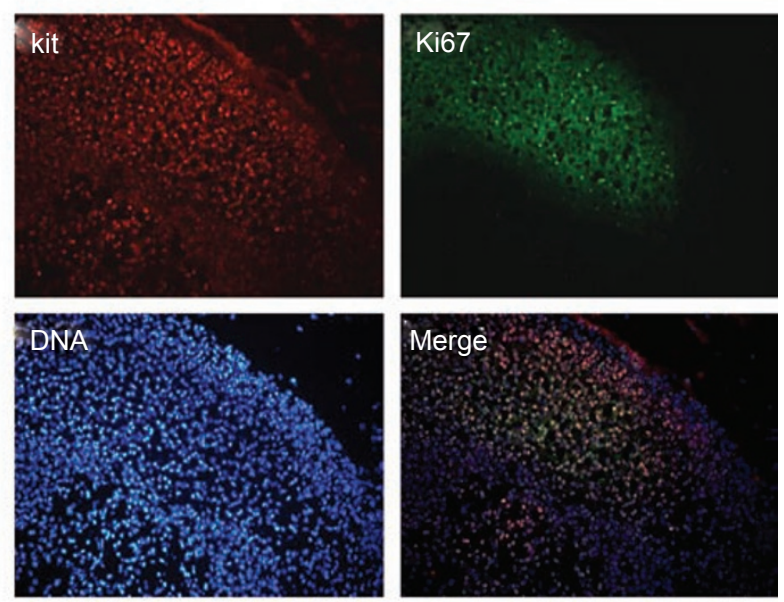

B
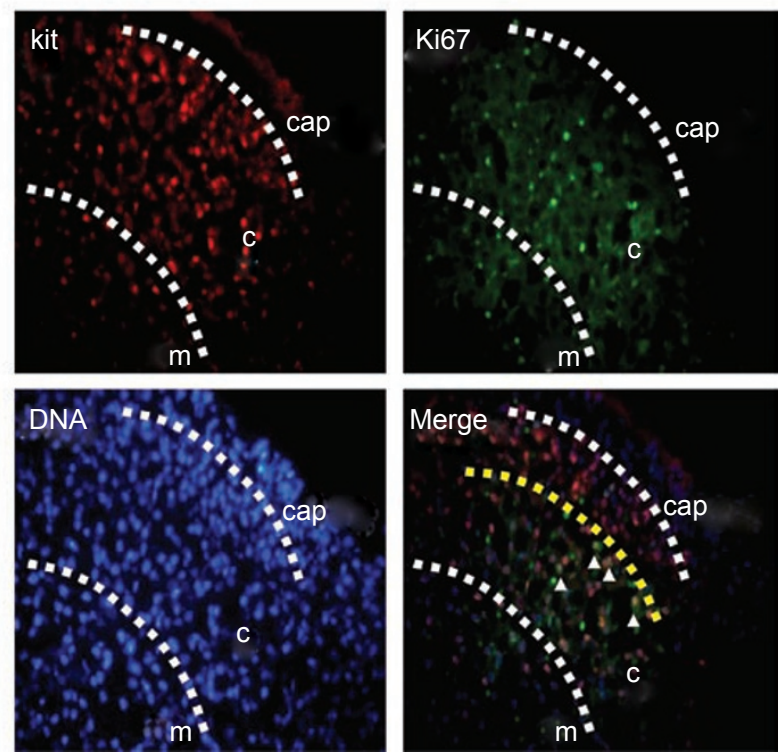

Figure 5 Microsections of c-kit and Ki67 expression in adrenal gland. Adrenal gland sections from WT mice were stained with specific antibodies for expression of Ki67 and c-kit. (A) Images at $20 \times$ magnification showing different regions of c-kit-positive and Ki67-positive cell distributions in the adrenal cortex of mice. (B) Images at $40 \times$ magnification showing the distinct zones of c-kit-positive cells in the outer region and Ki67-positive cells in the inner parenchyma of the adrenal cortex. Limited numbers of double-positive cells for c-kit and Ki67 expressions are indicated by arrowed. The regions of capsule (cap), cortex (c) and medulla (m) are delimited with white dot lines. The sectors of different c-kit ${ }^{+}$ and $\mathrm{Ki} 67^{+}$cell populations are bordered by a yellow dot line. liferating cells with differentiated features including gene expression of estrogen receptors.

\section{Discussion}

As a pleiotropic steroid hormone important in a variety of tissues, estrogen plays pivotal roles in tissue development and aging by regulating critical genes coding for cellular effectors. TERT is one important effector that is strictly controlled at transcriptional level. In man, TERT is expressed in stem cells, repressed in differentiated cells and reactivated in expanding neoplastic cells. In mice, telomerase is active in a number of tissues, underlying cell proliferation against aging [35]. The mechanisms of telomerase regulation under physiological conditions remain largely unknown. Using the estrogen-deficient mouse model, the present study shows for the first time that estrogen plays a key role in regulating telomerase activity in the adrenal gland of mice under physiological conditions. Our novel findings show that estrogen is essentially required for telomerase activity by up-regulating the TERT gene, and that estrogen deficiency causes telomerase inhibition and cell proliferation arrest in the adrenal gland, which could be reversed by 3 weeks of estrogen replacement therapy.

The findings of telomerase activity in the adrenal gland of mice and its inhibition by estrogen deficiency were surprising. While a very low level of telomerase activity has been detected in human and bovine adrenals, we found in this study that a significant level of telomerase activity is present in the adrenal gland of mice. The expression of telomerase activity in the mouse adrenal gland is further supported by the readily detectable gene expression of the telomerase catalytic subunit TERT. Our findings thus suggest that telomerase plays an important role in tissue developmental homeostasis of the adrenal gland in mice. The importance of telomerase activity in the adrenal gland is further reflected by the predominant regulation of the enzyme activity by estrogen. Our finding that estrogen withdrawal induces a dramatic inhibition of telomerase activity suggests an endocrine regulation of telomerase activity and thereby adrenal gland genesis and homeostasis. This significant regulation of telomerase activity by estrogen in the adrenal gland is in marked contrast to the lack of estrogen regulation of telomerase activity in other tissues such as liver, where there are high levels of telomerase activity. This tissue-specific effect of estrogen on telomerase activity suggests that diverse mechanisms exist in the regulation of telomerase activity and the actions of estrogen by tissue factors. By measuring telomerase activity in crude tissue extracts, we cannot exclude cell type-specific regulation of telomerase activity by estrogen in the tissues and organs examined in this study. It is pos- 
sible that the effect of estrogen can be masked or diluted with mixed cell populations, while steroid regulation of telomerase may occur in a cohort of the fractions. This may be relevant for further research, particularly in tissues such as the uterus and thymus, where estrogen plays fundamental roles in regulating cell proliferation in different compartments. In our recent studies in the ovarian tissue, we have found that estrogen regulates telomerase activity in the granulosa cells of ovarian follicles (manuscript submitted). Given that transplantation of adrenocortical cells expressing telomerase activity restores the adrenal hormone levels in adrenalectomized mice [36], it is likely that the adrenal gland preserves a population of telomerase-positive cells to retain cell proliferation capacity with differentiation for adequate function of the adrenal gland in mice.

Consistent with the hypothesis that estrogen regulates telomerase activity and thereby tissue genesis and repair in a tissue-specific manner, elimination of estrogen causes a significant decrease of telomerase activity in association with the loss of tissue weight of the adrenal gland. Examination of cell proliferation by immunofluorescent staining for cell proliferation-specific expression of Ki67 in the adrenal tissue sections shows that cell proliferative activity predominantly occurs in the adrenal cortex, whereas little cell proliferative activity is observed in the adrenal medulla consistent with the most differentiated neural-like cells residing in the medulla with repressed telomerase activity [37]. Moreover, in the absence of estrogen, the number of proliferating cells in the cortex has been significantly reduced as observed in the ArKO animals. Thus, estrogen regulation of cell proliferation and perhaps tissue genesis in the adrenal gland likely involves telomerase. Furthermore, we found that telomeres are longer in the cortex than in the medulla, and the length of telomeres in the adrenal cortex is dramatically reduced by more than $60 \%$ in the absence of estrogen in association with inhibited telomerase activity. Thus, our findings show that estrogen up-regulates telomerase activity and facilitates telomere maintenance and adrenal cortex cell proliferation in mice. This conclusion is consistent with our previous findings in human epithelial cells that estrogen stimulates telomerase activity and promotes cell survival [20], and is further supported by the experiments of hormone replacement therapy in which estrogen treatment restores telomerase activity, cell proliferation and tissue weight.

Previous studies have shown that oophorectomy results in atrophy of the adrenal gland in rat [38]. Our present study using the estrogen-deficient mouse model shows for the first time that removal of the estrogen results in a decrease of the mass of adrenal gland and cellular proliferative activity, suggesting that the atrophy of adrenal gland in oophorectomy is mediated by estrogen deficiency. The ac- companied decrease of telomerase activity and shortening of telomeres in the absence of estrogen further suggest that telomerase inhibition and telomere shortening are involved in mediating the effect of estrogen loss. The recovery of telomerase activity and cell proliferation following estrogen replacement therapy further supports a role of telomerase activity in mediating the stimulatory effect of estrogen on cell proliferation in the adrenal gland. Given that the cell proliferative activity in response to estrogen occurs primarily in the outer quarter of the adrenal cortex, a structural zone where stem cells have been localized and shown to proliferate and migrate centripetally to the proximity of the boundary with the adrenal medulla [27, 39], it is possible that estrogen up-regulations of TERT gene, telomerase activity, telomere maintenance and cell proliferation primarily occur in the stem cell or progenitor cell compartment of adrenal gland. This speculation is consistent with recent studies showing that telomerase regulates the maintenance of stem cell proliferative potential, stem cell mobilization, proliferation and differentiation for tissue regeneration and repair [40-42]. Our findings that there is a gradient distribution of cell populations of majority c-kit positive cells, small number of both c-kit and Ki67 positive cells, and majority Ki67 positive cells from the cortex toward the boundary of medulla suggest that $\mathrm{c}-\mathrm{kit}^{+}$cells of more stemness in the outer region undergo centripetal migration and differentiation to $\mathrm{c}-\mathrm{kit}^{+} / \mathrm{Ki} 67^{+}$progenitors and subsequently more differentiated $\mathrm{Ki} 67^{+}$proliferative population within the adrenal parenchyma. The limited co-localization of c-kit with Ki67 suggests a distinct population of Ki67positive proliferative cells affected by estrogen deficiency. This population may be differentiated progenitor cells with distinct gene expression characteristics including emerged estrogen receptors and Ki67 and vanishing c-kit.

The mechanisms of estrogen regulation of telomerase activity and thereby cell proliferation in the adrenal gland of mice have not been fully understood. The findings that the absence of estrogen is associated with a significant reduction of the telomerase catalytic subunit TERT and that estrogen replacement therapy restores TERT gene expression to WT levels suggest a cause-effect relationship between estrogen and telomerase. The data suggest that estrogen up-regulates telomerase activity by stimulating the transcription of TERT, consistent with previous reports that estrogen stimulates TERT gene promoter activity and gene transcription in cultured human vascular endothelial cells [20], ovary epithelium cells [21] and cancer cells [22]. While the adrenal cortex contains estrogen receptors $[43,44]$, the mouse TERT gene promoter region shows no consensus signature of an estrogen receptor binding site. It is therefore possible that estrogen regulates mouse TERT gene expression by indirect trans actions. This is sup- 
ported by our data that gene expression levels of c-myc, a transcription factor of TERT [17, 18, 45], are significantly reduced in ArKO adrenals and its expression levels are restored to the levels comparable with WT after estrogen replacement therapy. Estrogen has been shown to rapidly stimulate c-myc gene expression in ER-positive cell lines in $1 \mathrm{~h}$ of treatment [46], suggesting that c-myc is a direct downstream target gene of ER. Moreover, E2 stimulates telomerase activity in c-myc-positive human choriocarcinoma BeWo cells, but has no effect on telomerase activity in c-myc-null HO15.19 cells [47]. We cannot exclude other mechanisms including nuclear signalling from the membrane-associated ER $[48,49]$ and epigenetic control of the TERT gene [50] in the adrenal gland. Future studies should also consider potential impacts of estrogen deficiency on other factors involved in telomere homeostasis, including telomere binding proteins [51, 52].

In summary, we have identified a direct connection from estrogen to the telomerase gene TERT in vivo under physiological conditions in mouse adrenal gland. Maintaining the TERT gene expression by estrogen allows normal growth of the adrenal gland in mice. Deficiency of estrogen leads to inhibition of telomerase activity and cell proliferation. Hormone replacement therapy is sufficient to restore the lost telomerase activity at the level TERT gene transcription, and thereby reverse the compromised cell proliferation after 3 weeks of treatment.

\section{Materials and Methods}

\section{Mice}

ArKO mice were generated by targeted disruption of the Cyp 19 gene encoding aromatase that catalyzes the conversion of estrogen from androgen [53]. Mice were bred, genotyped and maintained as previously described [54]. A 21-day release E2 $(0.05 \mathrm{mg}$ E2 per pellet; Innovative Research of America) or placebo pellet was subcutaneously implanted into 10-12-week-old female WT and ArKO mice. Tissues were collected on the $22^{\text {nd }}$ day, weighed and processed as required.

\section{Telomerase activity assay}

Female WT and ArKO mice were killed by $\mathrm{CO}_{2}$ inhalation and tissues were snap frozen in liquid nitrogen and stored at $-80^{\circ} \mathrm{C}$ until use. A TRAP assay was performed as described previously [55] to determine telomerase activity. Frozen tissues were homogenized in 10 times volume of pre-chilled TRAP lysis buffer $(0.5 \%$ CHAPS, $10 \mathrm{mM}$ Tris- $\mathrm{HCl} \mathrm{pH} 7.5,1 \mathrm{mM} \mathrm{MgCl} 2,1 \mathrm{mM}$ EGTA, $5 \mathrm{mM}$ $\beta$-mercaptoethanol, $10 \%$ glycerol and $1 \mathrm{mM} \mathrm{AEBSF}$ ). Nuclei were pelleted by centrifugation at $3000 \times g$, resuspended in TRAP lysis buffer and nuclear proteins were isolated by centrifugation at $600 \times g$. Protein concentration was determined using a colorimetric protein assay kit (Bio-Rad, US). Equal amounts $(0.4 \mu \mathrm{g})$ of proteins were incubated at $30{ }^{\circ} \mathrm{C}$ for $20 \mathrm{~min}$ in a $30 \mu \mathrm{l}$ reaction containing $20 \mathrm{mM}$ Tris- $\mathrm{HCl} \mathrm{pH} \mathrm{8.3,} 1.5 \mathrm{mM} \mathrm{MgCl} 2,63 \mathrm{mM} \mathrm{KCl}, 0.05 \%$ Tween-20, $1 \mathrm{mM}$ EDTA, $2 \mu \mathrm{g}$ BSA, $50 \mu \mathrm{M}$ each dNTP and $0.1 \mu \mathrm{g}$ telomerase substrate. De novo synthesized telomeric repeats were amplified in the presence of $0.1 \mu \mathrm{g}$ ACX, $2 \mathrm{U}$ Taq DNA polymerase (GeneWorks) and $0.05 \mu \mathrm{Ci}\left[\alpha-{ }^{32} \mathrm{P}\right] \mathrm{dATP}$ (Perkin-Elmer) using the following PCR conditions: $94{ }^{\circ} \mathrm{C}$ for $3 \mathrm{~min}$, followed by 25 cycles of $94{ }^{\circ} \mathrm{C} 30 \mathrm{~s}$, $60{ }^{\circ} \mathrm{C} 30 \mathrm{~s}$ and $72{ }^{\circ} \mathrm{C} 45 \mathrm{~s}$. To monitor potential non-specific PCR effects, an additional primer pair of TSNT and NT was added to the reaction to give rise to an internal control (IC). Telomeric products were resolved on a nondenaturing polyacrylamide gel, followed by overnight autoradiography. Semi-quantitative analysis of telomerase activity was performed by counting ${ }^{32} \mathrm{P}$ activity using a $\beta$-radiation counter [55]. To control for telomerase activity, all assays included CHAPS lysis buffer as a negative control and HeLa cell lysates as a positive control.

\section{$R T-P C R$}

Total RNAs were extracted using a high pure RNA tissue extraction kit (Roche). Equal amounts of RNAs were reverse transcribed with oligodT(20) using the ThermoScript system (Invitrogen) according to the manufacturer's instructions. RT-PCR was used to measure gene expression of TERT and c-myc. Expression of each gene was normalized to the housekeeping gene GAPDH. The primers used were: TERT-F 5'-CTC TCT GCT GCG CAG CCC ATA C-3'; TERT-R 5'-CCT CGT TAA GCA GCT CAAAG-3'; c-myc-F 5' -TCA CTG GAA CTT ACAATC TGC GAG C-3'; c-myc-R 5' -TCC TGT TGG TGA AGT TCA CGT TGA G-3'; GAPDH-F 5'-CAG ATC CAC AAC GGA TAC ATT GGG-3'; GAPDH-R 5'-CAT GAC AAC TTT GGC ATT GTG G-3'. Experiments were conducted to ensure that amplification was within the logarithmic range. PCR products were analyzed on $1.5 \%$ agarose gels stained with ethidium bromide. Products were measured using densitometry and each gene expressed as a ratio to GAPDH expression.

\section{Ki67 staining for cell proliferation}

Five $\mu \mathrm{m}$ fresh-frozen sections were used to stain for the Ki67 antigen, a marker of cell proliferation. Sections were fixed in $4 \%$ paraformaldehyde $10 \%$ sucrose, washed once in phosphate-buffered saline (PBS) and blocked with 5\% bovine serum albumin/PBS (Sigma, Fraction V) for $30 \mathrm{~min}$. Sections were incubated with polyclonal rabbit anti-Ki67 (Abcam, No. ab15580) at $4{ }^{\circ} \mathrm{C}$ overnight at a 1:400 dilution. Primary antibody was detected with donkey antirabbit IgG-FITC (Santa Cruz Biotechnology, sc-2090) incubated for $90 \mathrm{~min}$ at room temperature at a 1:100 dilution. Nuclei were stained with $0.5 \mu \mathrm{g} / \mu \mathrm{l} \mathrm{Hoechst} 33258$ (Sigma) and visualized by fluorescence microscopy. Results were expressed as the number of Ki67-positive cells per gland \pm standard error.

\section{Telomere Q-FISH}

The slides of adrenal tissue sections were fixed in $4 \%$ formaldehyde before treatment with acidified $1 \%$ pepsin solution, and hybridized with probe solution $(0.3 \mu \mathrm{g} / \mathrm{ml} \mathrm{Cy} 3$-conjugated [CCCTAA]3 PNA probe (Panagene, Daejeon, South Korea), 70\% formamide, $20 \mathrm{mM}$ Tris- $\mathrm{HCl}, \mathrm{pH} 7.0,1 \% \mathrm{BSA})$. Washing was conducted in $\mathrm{PBS} /$ tween-20 with one high stringency wash at $57^{\circ} \mathrm{C}$. DNA was counterstained with DAPI and visualized and captured using Nikon Eclipse TE2000 microscope, Plan Fluor 40× objective, DS-5MC CCD camera and NIS-Elements F 2.20 software (Nikon). Telomere images were captured with a Plan Fluor $100 \times$ oil-emersion objective, and individual telomere fluorescence was integrated using spot IOD analysis in the TFL-Telo 2.2 program (gift from Dr Peter Lansdorp, 
Vancouver) [56]. Images from at least 13 metaphase spreads of each data point were quantified before assembly of data in a standard spreadsheet program. At least 50 nuclei from each condition were analyzed.

\section{Statistical analysis}

Data were analyzed using Student $t$-tests and a probability $(P)$ value of less than 0.05 was considered statistically significant.

\section{Acknowledgments}

We thank Peter Lansdorp for the TFL-Telo 2.2 software program for telomere analysis. This work was supported by grants from the National Health and Medical Research Council of Australia, Australia Research Council, and Cancer Council of Victoria, Australia. MEJ was the recipient of an RD Wright Career Development Award and SB was the recipient of an Australian Postgraduate Award.

\section{References}

1 Allain JE, Dagher I, Mahieu-Caputo D, et al. Immortalization of a primate bipotent epithelial liver stem cell. Proc Natl Acad Sci USA 2002; 99:3639-3644.

2 DeNardo DG, Kim H-T, Hilsenbeck S, et al. Global gene expression analysis of estrogen receptor transcription factor cross talk in breast cancer: identification of estrogen-induced/activator protein-1-dependent genes. Mol Endocrinol (Baltimore, MD) 2005; 19:362-378.

3 Cheng ASL, Jin VX, Fan M, et al. Combinatorial analysis of transcription factor partners reveals recruitment of c-MYC to estrogen receptor-[alpha] responsive promoters. Mol Cell 2006; 21:393-404.

4 Bayne S, Liu JP. Hormones and growth factors regulate telomerase activity in ageing and cancer. Mol Cell Endocrinol 2005; 240:11-22.

5 Bayne S, Jones ME, Li H, Liu JP. Potential roles for estrogen regulation of telomerase activity in aging. Ann N Y Acad Sci 2007; 1114:48-55.

6 Liu JP. Studies of the molecular mechanisms in the regulation of telomerase activity. FASEB J 1999; 13:2091-2104.

7 Liu JP, Deb S, Li H. Regulation of telomerase activity. In: Krupp G, Parwaresch R, eds. Telomeres and Telomerases: Cancer and Biology. New York: Landes Bioscience/Eurekah.com, 2002:134159.

8 Liu JP, Cassar L, Pinto A, Li H. Mechanisms of cell immortalization mediated by EB viral activation of telomerase in nasopharyngeal carcinoma. Cell Res 2006; 16:809-817.

9 Blackburn EH. Telomeres and telomerase: their mechanisms of action and the effects of altering their functions. FEBS Lett 2005; 579:859-862.

10 Blasco MA. Telomeres and human disease: ageing, cancer and beyond. Nat Rev Genet 2005; 6:611-622.

11 Hahn WC. Telomere and telomerase dynamics in human cells. Curr Mol Med 2005; 5:227-231.

12 Dwyer J, Li H, Xu D, Liu JP. Transcriptional regulation of telomerase activity: roles of the ets transcription factor family. Ann N Y Acad Sci 2007; 1114:36-47.
13 Li H, Liu JP. Mechanisms of action of TGF-beta in cancer: evidence for Smad3 as a repressor of the hTERT gene. Ann NY Acad Sci 2007; 1114:56-68.

14 Cong YS, Wen J, Bacchetti S. The human telomerase catalytic subunit hTERT: organization of the gene and characterization of the promoter. Hum Mol Genet 1999; 8:137-142.

15 Horikawa I, Cable PL, Mazur SJ, et al. Downstream E-boxmediated regulation of the human telomerase reverse transcriptase (hTERT) gene transcription: evidence for an endogenous mechanism of transcriptional repression. Mol Biol Cell 2002; 13:2585-2597.

16 Kyo S, Inoue M. Complex regulatory mechanisms of telomerase activity in normal and cancer cells: How can we apply them for cancer therapy? Oncogene 2002; 21:688-697.

$17 \mathrm{Wu}$ KJ, Grandori C, Amacker M, et al. Direct activation of TERT transcription by c-MYC. Nat Genet 1999; 21:220-224.

$18 \mathrm{Xu}$ D, Popov N, Hou M, et al. Switch from Myc/Max to Mad1/ Max binding and decrease in histone acetylation at the telomerase reverse transcriptase promoter during differentiation of HL60 cells. Proc Natl Acad Sci USA 2001; 98:3826-3831.

$19 \mathrm{Li} \mathrm{H}, \mathrm{Xu} \mathrm{D}$, Li J, et al. Transforming growth factor beta suppresses human telomerase reverse transcriptase (hTERT) by Smad3 interactions with c-Myc and the hTERT gene. $J$ Biol Chem 2006; 281:25588-25600.

20 Ling S, Zhou L, Li H, et al. Effects of 17beta-estradiol on growth and apoptosis in human vascular endothelial cells: influence of mechanical strain and tumor necrosis factor-alpha. Steroids 2006; 71:799-808.

21 Misiti S, Nanni S, Fontemaggi G, et al. Induction of hTERT expression and telomerase activity by estrogens in human ovary epithelium cells. Mol Cell Biol 2000; 20:3764-3771.

22 Kyo S, Takakura M, Kanaya T, et al. Estrogen activates telomerase. Cancer Res 1999; 59:5917-5921.

23 Chadeneau C, Siegel P, Harley CB, et al. Telomerase activity in normal and malignant murine tissues. Oncogene 1995; 11:893898.

24 Burger A, Double J. Telomerase activity in normal and malignant mammalian tissues: feasibility of telomerase as a target for chancer chemotherapy. Br J Cancer 1997; 75:516-522.

25 Prowse K, Greider C. Developmental and tissue-specific regulation of mouse telomerase and telomere length. Proc Natl Acad Sci USA 1995; 92:4818-4822.

26 Tischler AS, Powers JF, Shahsavari M, et al. Comparative studies of chromaffin cell proliferation in the adrenal medulla of rats and mice. Fundam Appl Toxicol 1997; 35:216-220.

27 Zajicek G, Ariel I, Arber N. The streaming adrenal cortex: direct evidence of centripetal migration of adrenocytes by estimation of cell turnover rate. J Endocrinol 1986; 111:477-482.

28 Verhofstad AA. Kinetics of adrenal medullary cells. J Anat 1993; 183:315-326.

29 Scholzen T, Gerdes J. The Ki-67 protein: from the known and the unknown. J Cell Physiol 2000; 182:311-322.

$30 \mathrm{Kim}$ AC, Hammer GD. Adrenocortical cells with stem/progenitor cell properties: recent advances. Mol Cell Endocrinol 2007; 265-266:10-16.

31 Barile L, Chimenti I, Gaetani R, et al. Cardiac stem cells: isolation, expansion and experimental use for myocardial regeneration. Nat Clin Pract Cardiovasc Med 2007; 4 Suppl 1:S9-S14.

32 Okada $\mathrm{S}$, Nakauchi $\mathrm{H}$, Nagayoshi $\mathrm{K}$, et al. In vivo and in vitro stem cell function of c-kit- and Sca-1-positive murine hemato- 
poietic cells. Blood 1992; 80:3044-3050.

33 Spangrude GJ, Heimfeld S, Weissman IL. Purification and characterization of mouse hematopoietic stem cells. Science 1988; 241:58-62.

34 Manova K, Huang EJ, Angeles M, et al. The expression pattern of the c-kit ligand in gonads of mice supports a role for the c-kit receptor in oocyte growth and in proliferation of spermatogonia. Dev Biol 1993; 157:85-99.

35 Blasco MA. Telomere length, stem cells and aging. Nat Chem Biol 2007; 3:640-649.

36 Huang Q, Chen M, Liang S, et al. Improving cell therapy - experiments using transplanted telomerase-immortalized cells in immunodeficient mice. Mech Ageing Dev 2007; 128:25-30.

37 Li H, Pinto AR, Duan W, et al. Telomerase down-regulation does not mediate PC12 pheochromocytoma cell differentiation induced by NGF, but requires MAP kinase signalling. J Neurochem 2005; 95:891-901.

38 Winter CA, Emery FE. Compensatory adrenal hypertrophy in the rat as influenced by sex, castration, time and thymectomy. Anat Rec 1936; 66:401-409.

39 Mitani F, Mukai K, Miyamoto H, et al. Development of functional zonation in the rat adrenal cortex. Endocrinology 1999; 140:3342-3353.

40 Flores I, Cayuela ML, Blasco MA. Effects of telomerase and telomere length on epidermal stem cell behaviour. Science 2005; 309:1253-1256.

41 Sarin KY, Cheung P, Gilison D, et al. Conditional telomerase induction causes proliferation of hair follicle stem cells. Nature 2005; 436:1048-1052.

42 Yamaguchi H, Calado RT, Ly H, et al. Mutations in TERT, the gene for telomerase reverse transcriptase, in aplastic anemia. $N$ Engl J Med 2005; 352:1413-1424.

43 Toda K, Hayashi Y, Okada T, et al. Expression of the estrogeninducible EGFP gene in aromatase-null mice reveals differential tissue responses to estrogenic compounds. Mol Cell Endocrinol 2005; 229:119-126.

44 Muller RE, Wotiz HH. Estrogen-binding protein in mouse and rat adrenal glands. J Biol Chem 1978; 253:740-745.

45 Greenberg RA, O'Hagan RC, Deng H, et al. Telomerase reverse transcriptase gene is a direct target of c-Myc but is not functionally equivalent in cellular transformation. Oncogene 1999; 18:1219-1226.

46 Santos GF, Scott GK, Lee WMF, et al. Estrogen-induced posttranscriptional modulation of c-myc proto-oncogene expression in human breast cancer cells. $J$ Biol Chem 1988; 263:95659568

47 Sarkar P, Shiizaki K, Yonemoto J, Sone H. Activation of telomerase in BeWo cells by estrogen and 2,3,7,8-tetrachlorodibenzo-p-dioxin in co-operation with c-Myc. Int J Oncol 2006; 28:43-51.

48 Revankar CM, Cimino DF, Sklar LA, et al. A transmembrane intracellular estrogen receptor mediates rapid cell signaling. Science 2005; 307:1625-1630.

49 Segars J, Driggers P. Estrogen action and cytoplasmic signaling cascades. Part I: Membrane-associated signaling complexes. Trends Endocrinol Metab 2002; 13:349-354.

50 Liu C, Fang X, Ge Z, et al. The telomerase reverse transcriptase (hTERT) gene is a direct target of the histone methyltransferase SMYD3. Cancer Res 2007; 67:2626-2631.

51 Zhang DH, Zhou B, Huang Y, et al. The human Pif1 helicase, a potential Escherichia coli RecD homologue, inhibits telomerase activity. Nucleic Acids Res 2006; 34:1393-1404.

52 Xin H, Liu D, Wan M, et al. TPP1 is a homologue of ciliate TEBP-beta and interacts with POT1 to recruit telomerase. Nature 2007; 445:559-562.

53 Fisher CR, Graves KH, Parlow AF, Simpson ER. Characterization of mice deficient in aromatase (ArKO) because of targeted disruption of the cyp19 gene. Proc Natl Acad Sci USA 1998; 95:6965-6970.

54 Jones M, Thorburn A, Britt K, et al. Aromatase-deficient (ArKO) mice have a phenotype of increased adiposity. Proc Natl Acad Sci USA 2000; 97:12735-12740.

55 Li H, Zhao LL, Funder JW, Liu JP. Protein phosphatase 2A inhibits nuclear telomerase activity in human breast cancer cells. J Biol Chem 1997; 272:16729-16732.

56 Rufer N, Dragowska W, Thornbury G, et al. Telomere length dynamics in human lymphocyte subpopulations measured by flow cytometry. Nat Biotechnol 1998; 16:743-747. 